\title{
Salt-Sensitive Hypertension: Can We Convert Salt-Sensitive State to a Salt- Resistant One?
}

\section{Mohamed G. Atta*}

Department of Medicine, Johns Hopkins University School of Medicine, Baltimore, Maryland, USA

Hypertension is a complex condition and a full understanding of its pathophysiology is unsatisfactory. It's often considered to be a state of too much salt or too much renin, with overlap between these two mechanisms possible.

This basic salt-versus-renin distinction has imposed, for the most part, approach to treatment-lower dietary sodium plus diuretics for patients with excessive salt (low-renin or salt-sensitive hypertension), and inhibition of the renin-angiotensin-aldosterone system for patients with high-renin hypertension (salt-resistant hypertension). But the potential role of the lymphatic network in hypertension has been overlooked.

\section{Low-Renin Hypertension}

The hypothesis for the development of low-renin hypertension, which is driven by increased salt retention, proposes a causal relationship between salt intake, total body sodium and blood pressure.

In this model, the decrease in renal excretion of sodium is associated with a subsequent increase in extracellular volume and vascular resistance, resulting in hypertension. Supporting this hypothesis is monogenic form of hypertension, where mutations in renal ion transporters are associated with volume expansion. The extracellular volume in the model is distributed between the intravascular and the interstitium compartments. Sodium retention elicits water retention and an isotonic environment between the two compartments is maintained.

\section{The Three-Compartment Model}

This two-compartment model has been challenged in recent years by Machnik and colleagues [1]. In their elegant work, those investigators discovered that sodium accumulation is not necessarily associated with water retention. Instead, high-salt diet in rats led to hypertonic water-free sodium accumulation in the interstitium of the skin. This accumulation resulted in increased density and hyperplasia in the lymph capillary network.

The mechanisms that trigger these effects on the lymphatics are like those utilized by the renal medullary and brain cells in response to a hypertonic environment: Hypertonicity, which causes cell to shrink, elicits specific processes that restore cell volume, allowing normal cellular functions to proceed.

Similarly, in salt-sensitive hypertension, the accumulation of sodium prompts an activation of tonicity-responsive enhancer binding protein (TonEBP) in mononuclear phagocyte system (MPS) cells in the interstitium of the skin.

Signaling within MPS derived-cells, which include macrophages and dendritic cells, leads to an increase in the activity of vascular endothelial growth factor-C (VEGF-C), the development of hyperplasia in the lymph capillary network and an increase in the expression of endothelial nitric oxide synthase. This pathway helps to regulate volume and blood pressure, inhibiting it by blocking VEGF-C or depleting macrophage scan results in hypertension.

MPS cells provide a buffering mechanism for hypertension. Through VEGF-C-mediated hyperplasia in the preexisting lymph capillary network, the lymphatics expand. This expansion provides an internal means of sodium clearance and interstitial electrolyte and volume homeostasisis maintained.

This extra-renal mechanism of sodium homeostasis suggests, as indicated by Machnik and colleagues, a dynamic three-, rather than two, compartment model, where the interstitium is a separate, locally regulated space. In this model, failure of this physiological extra-renal regulatory mechanism leads to a salt-sensitive blood pressure response. This indicates that depletion of the MPS cells or blockade of VEGF-C regulatory response can alter a salt-resistant blood-pressure state to a salt-sensitive state.

Of interest, the authors also found that plasma VEGF-C concentration is significantly elevated in patients with refractory hypertension. Unfortunately, lymphatic capillary hyperplasia and endothelial nitric oxide synthase expression were not evaluated in these individuals.

Although those findings do not invalidate the concept of pressure natriuresis and renal regulatory functions, it suggests that extracellular sodium, volume and blood pressure homeostasis cannot be maintained without extra-renal regulatory mechanisms.

\section{Clinical Implications}

Do patients with low-renin/salt-sensitivity hypertension have a dysfunctional lymphatic network or impaired MPS cell signaling? The significant increase in plasma VEGF-C concentration in patients with refractory hypertension is interesting and warrants further studies.

In clinical practice, agents that block VEGF signaling in cancer treatment have been associated with the development of hypertension. If these agents do in fact play a role in the condition, targeting the lymphatic network may provide a more specific and effective management strategy for low-renin hypertension than using diuretics.

Many medical organizations have endorsed diuretic use in the

${ }^{*}$ Corresponding author: Mohamed G. Atta, Department of Medicine, Johns Hopkins University School of Medicine, Baltimore, Maryland, USA, Tel: 410- 955-5268 ; Fax : 410- 955-0485; E-mail: matta1@jhmi.edu

Received January 28, 2012; Accepted January 30, 2012; Published February 01,2012

Citation: Atta MG (2012) Salt-Sensitive Hypertension: Can We Convert SaltSensitive State to a Salt-Resistant One? J Nephrol Therapeutic 2:e104 doi:10.4172/2161-0959.1000e104

Copyright: (C) 2012 Atta MG. This is an open-access article distributed under the terms of the Creative Commons Attribution License, which permits unrestricted use, distribution, and reproduction in any medium, provided the original author and source are credited. 
treatment of hypertension, including the Joint National Committee on Prevention, Detection, Evaluation and Treatment of High Blood Pressure. Diuretics also have been the primary intervention employed by investigators of several large randomized trials in hypertension, such as the ALLHAT (Antihypertensive and Lipid-Lowering Treatment to Prevent Heart Attack Trial) [2].

The side effect profile of diuretics, however, has often been ignored or overlooked by diuretic enthusiasts. This is due, in part, to the low cost of diuretics as compared with newer antihypertensive agents.

Given my own personal and scientifically biased view, which has largely been an "anti-diuretic" one, I welcome the work that may ultimately lead to the development of agents that promote extra-renal sodium clearance by inducing hypertonic sodium storage in the skin and ultimately converting salt-sensitive state to a salt-resistant one!

\section{References}

1. Machnik A, Neuhofer W, Jantsch J, Dahlmann A, Tammela T, et al. (2009) Macrophages regulate salt-dependent volume and blood pressure by a vascular endothelial growth factor-C-dependent buffering mechanism. Nat Med 15: 545-552.

2. Officers TA, Group CftACR (2002) Major Outcomes in High-Risk Hypertensive Patients Randomized to Angiotensin-Converting Enzyme Inhibitor or Calcium Channel Blocker vs Diuretic. JAMA 288: 2981-2997. 